\title{
Isolation and Expression Profiling of a CONSTANS-Like Gene and Two FLOWERING LOCUS T-Like Genes from Spinacia oleracea L.
}

\author{
Erika Abe', Kaien Fujino², Kiyoshi Masuda², Yube Yamaguchi'2,3* \\ ${ }^{1}$ Graduate School of Agriculture, Hokkaido University, Sapporo, Japan \\ ${ }^{2}$ Research Faculty of Agriculture, Hokkaido University, Sapporo, Japan \\ ${ }^{3}$ Graduate School of Life and Environmental Sciences, Osaka Prefecture University, Sakai, Japan \\ Email: ‘yu-yama@plant.osakafu-u.ac.jp
}

Received 17 November 2014; revised 5 December 2014; accepted 21 December 2014

Copyright @ 2014 by authors and Scientific Research Publishing Inc.

This work is licensed under the Creative Commons Attribution International License (CC BY).

http://creativecommons.org/licenses/by/4.0/

c) (i) Open Access

\begin{abstract}
Spinach (Spinacia oleracea L.) develops leaf rosettes under short-day conditions, and starts reproductive growth including bolting and flowering under long-day conditions. Japanese people prefer Oriental spinach that bolts easily with a shorter photoperiod than European spinach. This is one of the main reasons that Oriental spinach is difficult to grow year-round. In order to understand spinach flowering mechanisms and obtain knowledge for spinach breeding, we isolated one CONSTANS-like (COL) and two FLOWERING LOCUS T (FT) homologs, which are key components of photoperiodic regulation of flowering time, from a Japanese cultivar. The expression of SoCOL1 showed diurnal rhythm with the highest expression at the end of the dark cycle. This diurnal rhythm is similar to the expression of BvCOL1 from sugar beet (Beta vulgaris), whose flowerpromoting effect was observed when overexpressed in Arabidopsis. Phylogenetic analysis showed that SoCOL1 is the closest homolog of BvCOL1, suggesting that SoCOL1 is an ortholog of BvCOL1. SoFT1 and SoFT2 are closely related to BvFT1 and BvFT2, respectively. The expression of SoFT1 and SoFT2 were induced in advance of flower bud formation after changing the photoperiod, but the expression level of SoFT1 was much lower than SoFT2. Currently, we are speculating that SoFT2 is a flower-promoting factor of spinach, and that SoFT1 has a role in light signaling because the expression of SoFT1 showed a diurnal rhythm.
\end{abstract}

\section{Keywords}

CONSTANS, FLOWERING LOCUS T, Spinacia oleracea L., Flower Bud Formation, Photoperiod

\footnotetext{
*Corresponding author.
}

How to cite this paper: Abe, E., Fujino, K., Masuda, K. and Yamaguchi, Y. (2014) Isolation and Expression Profiling of a CONSTANS-Like Gene and Two FLOWERING LOCUS T-Like Genes from Spinacia oleracea L. American Journal of Plant Sciences, 5, 4018-4028. http://dx.doi.org/10.4236/ajps.2014.526420 


\section{Introduction}

Spinach (Spinacia oleracea L.) is a long-day plant, eaten at its vegetative growth stage, and one of the most popular vegetables in the world. After transition to the reproductive stage with flowering and bolting, spinach loses its value as an agricultural product, becoming bitter and hard. Spinach is thought to have originated in the Middle East and spread and developed in Europe (European spinach) and East Asia (Oriental spinach) [1]. Oriental spinaches, including Japanese cultivars, are less bitter than European spinach, having long petioles with a light crunchy texture and making them suitable for Japanese food preparation methods, such as brief boiling. However, Oriental spinaches flower early, requiring only a 12- to 13-hour-photoperiod, and can be grown only from autumn to winter. In contrast, European spinaches require a longer (14- to 16-hour-) photoperiod for flowering and have a bitter taste compared to Oriental spinaches, being more suitable for western-style foods such as sautés. Although hybrid European and Oriental spinaches are used to provide spinach all year round in Japan, Oriental spinaches that require a longer photoperiod for flowering and bolting would be economically advantageous. In order to develop such spinach cultivars, it is necessary to understand flowering and bolting mechanisms of spinach at the genetic level.

In Arabidopsis, flowering and bolting are regulated by four major pathways (photoperiod, vernalization, GA-dependent and autonomous) [2] [3]. The major genes in the photoperiodic pathway are CONSTANS (CO), flowering integrator FLOWERING LOCUS T (FT) and TWIN SISTEROF FT (TSF) that control the floral transition in response to changing day length. $\mathrm{CO}$ belongs to a family of zinc finger transcription factors unique to plants [4]-[6]. CO expression exhibits a diurnal rhythm that is regulated by the circadian clock. CO protein is produced under a long-day photoperiod and activates FT and TSF transcription [7]. FT and TSF are members of the phosphatidylethanolamine-binding protein (PEBP) gene family, found in both angiosperms and gymnosperms. FT and TSF genes expressed in the leaf and hypocotyl vasculature and their proteins move to the shoot apical meristem (SAM) [8]. At the shoot apical meristem, FT and TSF form a complex with FLOWERING LOCUS D (FD), and upregulate the floral meristem identity gene APETALA1 to develop flower buds [9]. Generally, FT function is conserved among many plant species, but FT repressors vary widely among plant species. For example, FT and TSF belonging to the FT-like clade of the PEBP family act as floral integrators, and are repressed by TERMINAL FLOWER 1 (TFL1) [10], which belongs to the CEN/TFL-clade, another clade within PEBP family. In sugar beet, BvFT2 is thought to be an FT ortholog, and its function is repressed by BvFT1, which is the closest homolog of BvFT2 in the same FT-like clade [11].

Although regulations of spinach flowering and bolting by long-day photoperiods and gibberellin have been reported [12]-[16], detailed mechanisms at the genetic level have not yet been elucidated, especially within the photoperiodic pathway. Therefore, in this study we isolated CO and FT homologs from Oriental spinach ("Nihon”), and characterized them by expression analysis under different conditions.

\section{Materials and Methods}

\subsection{Plant Materials and Growth Conditions}

Spinach (Spinacia oleracea L., cv. "Nihon”, Sakata Seed, Japan) seeds were sown on an wet filter paper in a petri dish, incubated in the dark at $4^{\circ} \mathrm{C}$ until germination, and then transferred to pots containing a mixture of soil:vermiculite (1:1). All plants used in this study were cultivated in growth chambers at $22^{\circ} \mathrm{C}$ in long-day (LD) conditions (16 h light/8 h dark) or short-day (SD) conditions (9 h light/15 h dark). SD- and LD-grown spinach seedlings at the same leaf stage were used for gene expression analysis.

\subsection{Microscopic Observation of Spinach Shoot Apex}

In order to confirm whether spinach seedlings had develop flower buds at the microscopic level, spinach shoot apexes were harvested at each leaf stage, and fixed with FAA (Formalin: Acetic acid: Ethyl-alcohol: $\mathrm{H}_{2} \mathrm{O}=$ 10:5:50:35 v/v/v/v). The fixed samples were dehydrated using a graded series of ethyl-alcohol and n-butylalcohol solutions, and then embedded in paraffin (PARAPLAST PLUS Tissue Embedding Medium, McCormik Scientific, US). Microtome sections (eight $\mu \mathrm{m})$ were stained with $0.5 \%(\mathrm{w} / \mathrm{v})$ Safranine and $0.5 \%(\mathrm{w} / \mathrm{v})$ Fast Green FCF solution. Microscopic observation was performed using the Nikon ECLIPSE E600 (Nikon, Japan). 


\section{3. cDNA Synthesis}

Total RNAs were extracted from each tissue (cotyledons, leaves, shoot apexes and shoots) by TriPure Isolation Reagent (Roche). cDNA was synthesized from 500 ng total RNA with ReverTra Ace qPCR RT Master Mix with gDNA remover kit (TOYOBO) according to the manufacturer's protocol, and then diluted five times with TE buffer for further utilization.

\subsection{Isolation of FT and CO Homologs from Spinach}

Internal cDNA fragments of $F T$ and $C O$ were amplified using the BIOTAQ ${ }^{\mathrm{TM}} \mathrm{HS}$ polymerase PCR kit (BIOLINE) and degenerate primers (Table 1), which were designed from a highly conserved region of either FT or COL of Beta vulgaris (sugar beet) [11] [17] and Chenopodium rubrum [18] [19]. PCR products were cloned into the pGEM-T Easy vector (promega), and sequenced by a 3130 Genetic Analyzer (Applied Biosystems). Full-length cDNAs of $F T$ and $C O L$ homologs were obtained using Exact START ${ }^{\mathrm{TM}}$ Eukaryotic mRNA 5'- \& 3'-RACE Kit (Epiciente) with specific primers (Table 1) and Prime STAR GXL DNA Polymerase (TAKARA), cloned into pBlue Script II SK(-) vector (Agilent Technologies), and sequenced by 3130 Genetic Analyzer

Table 1. Primers used in this study.

\begin{tabular}{|c|c|}
\hline Name & Sequence (5’-3’) \\
\hline SoFT- $1^{1^{*}}$ & AGGYGATGACCTTAGGACTT \\
\hline SoFT- $2^{1^{*}}$ & CCTCCCTTTGRCAATTGAARTA \\
\hline SoFT1- $1^{1}$ & TAACTAGAGACTTTGCTGAACT \\
\hline SoFT1- $2^{1}$ & AGAGTTCAGCAAAGTCTCTAGT \\
\hline SoFT1- $3^{1}$ & TCATAGCAAACCACCTCTTGA \\
\hline SoFT2- $1^{1}$ & CAGTTGGAATTCATCGTTTCA \\
\hline SoFT2-2 $2^{1}$ & TCCAACTGARGGTCTTGGGT \\
\hline SoFT2-3 ${ }^{1^{*}}$ & TAGACYGAAGCAACGGGTAAA \\
\hline SoCO- $1^{1^{*}}$ & TGACACRTGCCGTKCAGCACC \\
\hline SoCO- $2^{1}$ & TGTGCTACCTGTGATGCTGA \\
\hline SoCO- $3^{1^{*}}$ & TCMACATCTGTTCTYTTWGCRAA \\
\hline SoCO- $4^{1}$ & TGAAGGGGATGAGGATGAAG \\
\hline SoCO- $5^{1}$ & СТТСАТССТСАТССССТТСА \\
\hline SoCO- $6^{1}$ & ACTGAATTCCTGCTGCTGGT \\
\hline SoFT1full-F ${ }^{1}$ & GCAACTTCTTAGTAATCCTACAATACC \\
\hline SoFT1full- $\mathrm{R}^{1}$ & AAAGAGAATCACTAGTTTTATTCAAGC \\
\hline SoFT2full-F ${ }^{1}$ & AАATTATTTTCTТААСССАТТСССТА \\
\hline SoFT2full-R ${ }^{1}$ & CGGTTTCTTAGACGGTAGAGTGA \\
\hline SoCOLfull-F $F^{1}$ & GAGAGAGAGATGATGAAGAAGGAA \\
\hline SoCOLfull-R ${ }^{1}$ & ACCGCACACTCAАСТCСАTT \\
\hline PCR primer $1^{2}$ & TCATACACATACGATTTAGGTGACACTATAGAGCGGCCGCCTGCAGGAAA \\
\hline PCR primer $2^{2}$ & TAGACTTAGAAATTAATACGACTCTATAGGCGCGCCACCG \\
\hline SoFT1- $\mathrm{a}^{3}$ & GGCCATCTGAAGTTGTTAGC \\
\hline SoFT1-b ${ }^{3}$ & AGACAGCAGCAACTGGTAGT \\
\hline SoFT2-a ${ }^{3}$ & AGGCCATCTGAGGTTGTTAAT \\
\hline SoFT2-b ${ }^{3}$ & AGTCTCGAGTGTTGAAGTTCT \\
\hline SoCO-a ${ }^{3}$ & TGAAGGGGATGAGGATGAAG \\
\hline SoCO-b ${ }^{3}$ & TCACATTCCATTCCGAACAA \\
\hline SoActin $\mathrm{F}^{3}$ & GGTGATGGTGTTAGTCACAC \\
\hline SoActin $\mathrm{R}^{3}$ & AATGATGGCTGGAAGAGAAC \\
\hline So $\alpha$ TUB-a ${ }^{3}$ & TGTTGTTCCCAAGGATGTGA \\
\hline So $\alpha$ TUB-b ${ }^{3}$ & GGCCCTCTTGGCATACATAA \\
\hline
\end{tabular}

${ }^{1}$ For isolation of spinach homologs. ${ }^{2}$ For 5'- and 3'-RACE kit primer (Epiciente). ${ }^{3}$ For qRT-PCR analysis. ${ }^{*}$ degenerate primer. 
(Applied Biosystems). Spinach FT and COL homologs were compared with other PEBP gene family members and CONSTANS-like gene family members, respectively, from several plant species. Multiple alignments were generated by the ClustalW program through the BioEdit program (https://www.bioedit.com/). Phylogenetic relationships of the PEBP and CTT domains of the PEBP gene family and CONSTANS-like gene family, respectively, were analyzed by the PHILIP program with SEQBOOT, PROTPARS, and CONSENSE programs (PHYLIP 3.68; http://evolution.genetics.washington.edu/phylip.html), and the phylogenetic tree was visualized by the TreeView program (http://taxonomy.zoology.gla.ac.uk/rod/treeview.html).

\subsection{Reverse Transcript Quantitative-PCR (RT-qPCR)}

Synthesized cDNA was subjected to quantitative-PCR analysis using the CFX Connect ${ }^{\mathrm{TM}}$ Real-Time System (Bio-Rad) with SYBR green. Reaction mixture contained $10 \mu$ of THUNDERBIRD ${ }^{\circledR}$ SYBR ${ }^{\circledR}$ qPCR Mix (TOYOBO), $2 \mu \mathrm{l}$ of cDNA solution, and $200 \mathrm{nM}$ gene-specific primers (Table 1) in a final volume of $20 \mu 1$. PCR conditions were: $95^{\circ} \mathrm{C}$ for $30 \mathrm{~s}$, followed by 45 cycles of $95^{\circ} \mathrm{C}$ for $10 \mathrm{~s}$ and $62^{\circ} \mathrm{C}$ for $30 \mathrm{~s}$. SoActin [14] and So $\alpha$ TUB [20] were amplified as internal standards.

\section{Results}

\subsection{Isolation of CO-Like and FT Homologs from Spinach}

Many studies have reported that FT-like genes and CONSTANS-like genes play crucial roles in flowering in various plant species [2]-[7]. However, the control mechanisms of expression of CONSTANS-like genes and FT-like genes are very complicated and vary among plant species. As the first step in clarification of spinach flowering mechanisms, we isolated two FT homologs and one COL homolog, which were designated as SoFT1, SoFT2 and SoCOL1, respectively, through CDNA amplification of highly conserved regions by degenerate primers followed by 5'- and 3'-RACE.

SoCOL1 protein showed 89\%, 92\%, 84\%, 54\%, 55\%, 60\% amino acid identities with BvCOL1 [17], CrCOL1, CrCOL2 [19], AtCO, AtCOL1 and AtCOL2 [5], respectively (Figure 1(a)). CO-like proteins are differentiated by the presence of either one or two zinc finger B-box domains in the $\mathrm{N}$-terminus of the protein and a C-terminal CCT domain, and are classified into three groups according to their B-box domain composition [4]-[6]. Type I CO-like proteins have two B-box domains; type II CO-like proteins have one B-box domain; and Type III CO-like proteins have one full B-box and one degraded B-box. The SoCOL1 protein has two B-box domains like the AtCO and BvCOL1 proteins, suggesting that SoCOL1 should be classified as a Type I CO-like protein. The resulting phylogenetic tree, which showed protein sequence relationships among CO family members, was consistent with our classification from the B-box composition (Figure 1(b)).

SoFT1 and SoFT2 proteins were highly similar to each other with $80 \%$ amino acid identities. Compared to FT homologs from other plant species, SoFT1 showed 74\%, 83\%, 83\%, 66\% and 74\% amino acid identity with BvFT1, BvFT2 [11], CrFT1, CrFT2 [18] and AtFT [10], respectively. SoFT2 showed 79\%, 89\%, 87\%, 60\% and 74\% amino acid identity with BvFT1, BvFT2, CrFT1, CrFT2 and AtFT, respectively (Figure 2(a)). The PEBP gene family is conserved among many plant species, and divided into three major clades, FT-like, CEN/ TFL-like and MFT-like clades. Phylogenetic analysis confirmed that SoFT1 and SoFT2 belong to the FT-like clade together with BvFT1, BvFT2 and AtFT (Figure 2(b)). Functionally important amino acid residues are mostly conserved in SoFT1 and SoFT2, but Gln-140 in AtFT is substituted for Pro in SoFT1 (Figure 2(a)) [21]-[23]. The substitution at this position was found only in CrFT2, whose activity as an FT has not been confirmed yet [18]. Interestingly, SoFT1 and CrFT2 have conserved Tyr-134, Gly-137 and Trp-138, which are important residues in BvFT2 for flower-promoting activity, although SoFT1 and CrFT2 are the closest homologs of BvFT1, a floral repressor of sugar beet with substitution of these three amino acid residues [11].

\subsection{Diurnal Rhythms of SoCOL1, SoFT1 and SoFT2 Expression}

The expression of $F T$ and $C O L$ from many plant species are regulated differentially by light and exhibit a diurnal rhythm [2]-[4]. It is necessary to investigate whether the expression of isolated genes are regulated by light or not. Spinach seedlings were grown in LD (16 h light/8 h dark) or SD (9 h light/15 h dark) conditions, and subjected to quantitative RT-PCR analysis when the seedlings were developing 3 - 4 leaves (Figure 3). At this leaf stage, seedlings grown in LD conditions have developed flower buds at the shoot apex, but those in SD conditions do not. SoCOL1 transcript accumulated after entering the dark period with the highest level at the end 

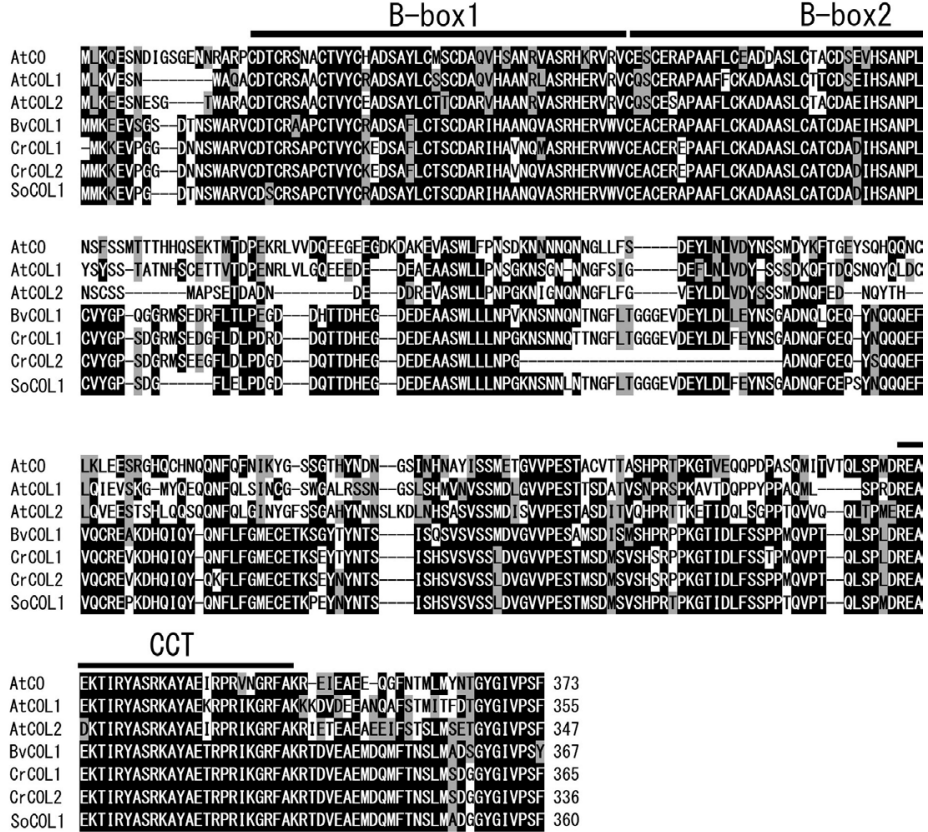

(a)

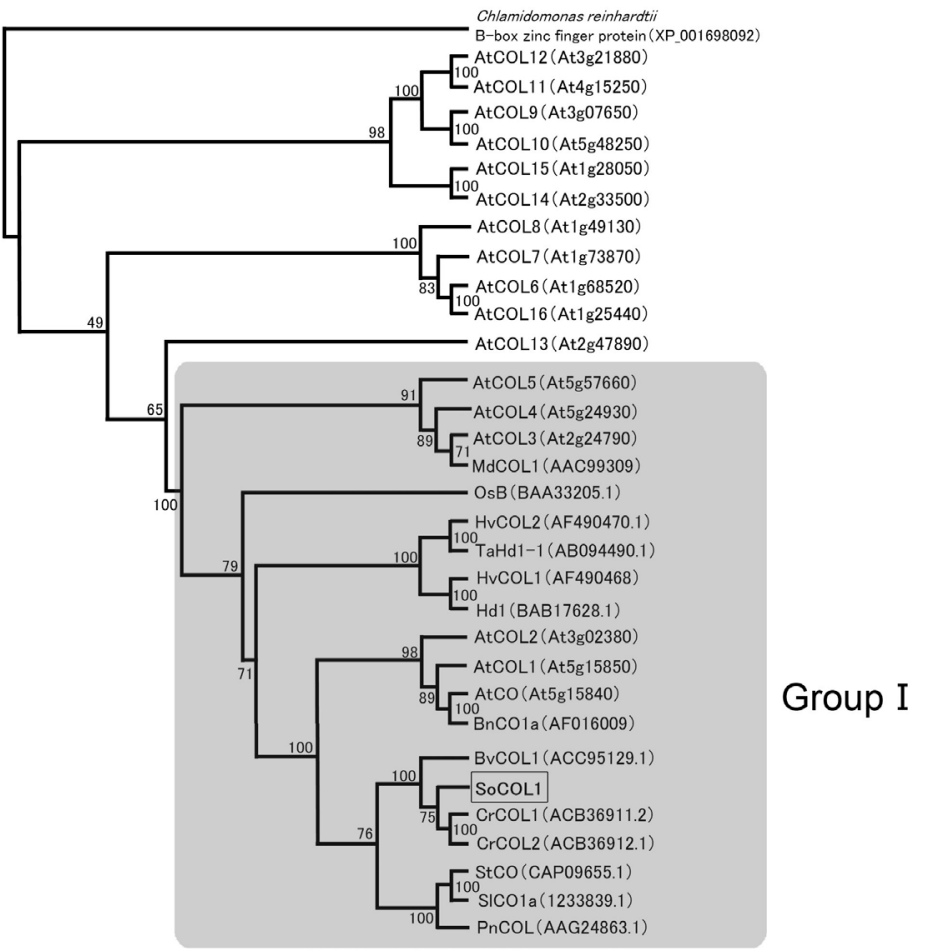

(b)

Figure 1. Comparison of amino acid sequence of SoCOL1 with COL gene family members from various plants. (a) Amino acid alignment of SoCOL1 with group I members of CONSTANS-like gene family from Arabidopsis, Sugar beet and Chenopodium rubrum. Domains characteristic of the CONSTANS-like gene family are indicated by black bars. (b) Phylogenetic analysis using protein sequences of COL family members from various plant species. Chlamydomonas B-box zinc finger protein was used as the outgroup. The numbers indicate bootstrap values. Protein sequences were obtained from GenBank. 


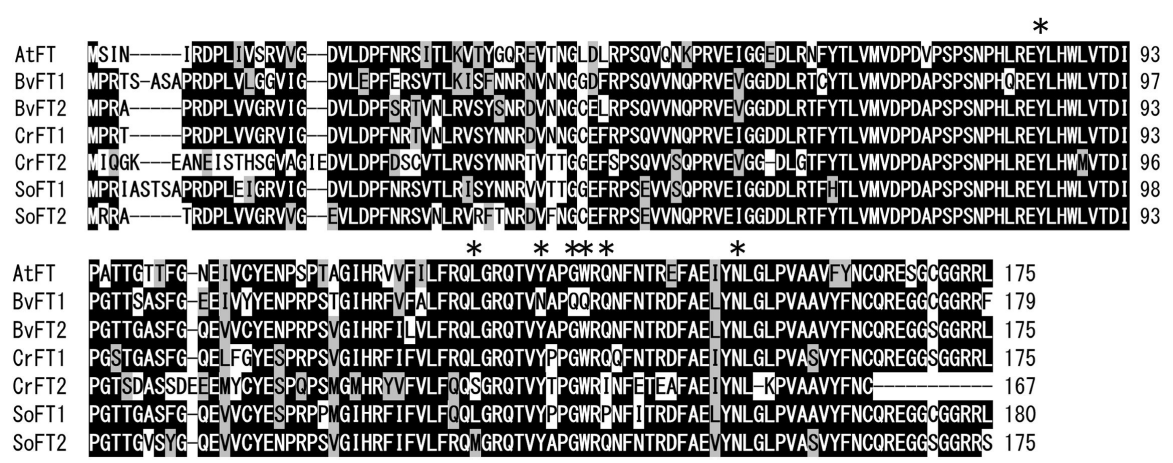

(a)

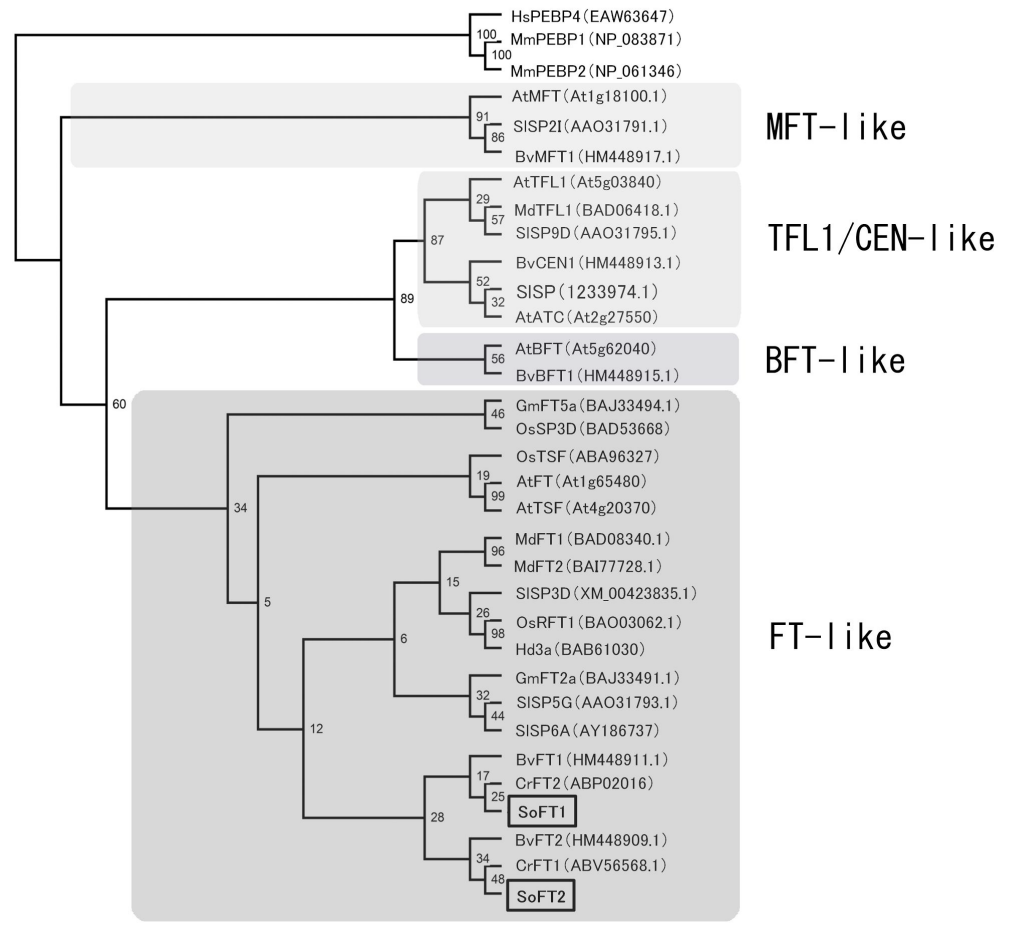

(b)

Figure 2. Comparison of amino acid sequences of SoFT1 and SoFT2 with other PEBP gene family members of various plants. (a) Amino acid alignment of SoFT1 and SoFT2 with FTs from Arabidopsis, Sugar beet and Chenopodium rubrum. Important amino acids conserved among FT-like clade are indicated by asterisks. (b) Phylogenetic analysis using protein sequences. PEBPs from Human (HsPEBP) and mouse (MmPEBP1 and MmPEBP2) were used as the outgroup. The numbers indicate bootstrap values. Protein sequences were obtained from GenBank.

of the dark period (zeitgeber time $[\mathrm{ZT}]=0$ ) in both LD and SD (Figure 3(a)). Despite the length difference of darkness, the maximum level of SoCOL1 expression is similar in LD and SD conditions, suggesting that there is some limitation for accumulation or that the induction is more efficient in LD conditions.

SoFT1 and SoFT2 showed much higher expression levels in seedlings grown in LD than in SD. Expression of SoFT1 of LD grown seedlings also had a diurnal rhythm with the highest expression at the end of the photoperiod (Figure 3(b)), while expression of SoFT2 did not show a clear diurnal rhythm (Figure 3(c)). These results suggested that expression of SoFT1 and SoFT2 were regulated by different pathways.

\subsection{Expression Level of SoFT1 and SoFT2 in Tissues}

Generally, FT mRNA is expressed in leaf vasculature, and the FT protein is transported to the SAM [2]. To 


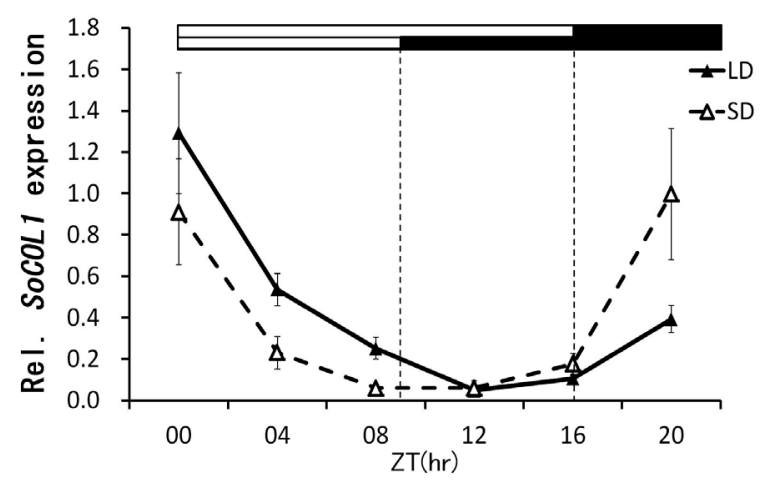

(a)

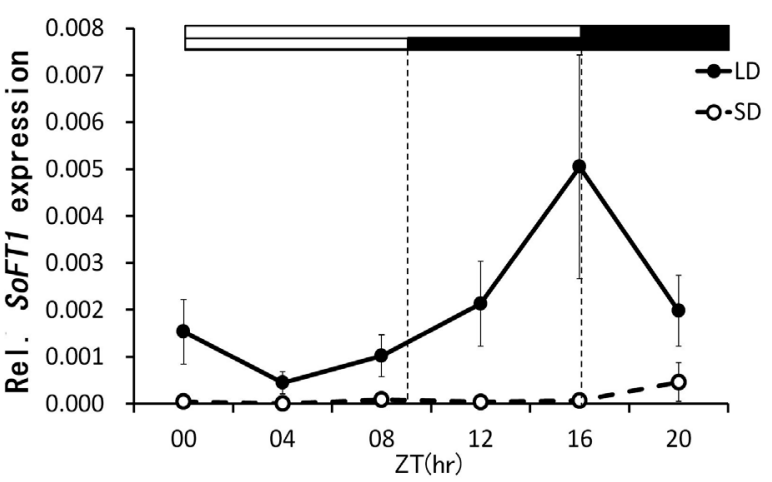

(b)

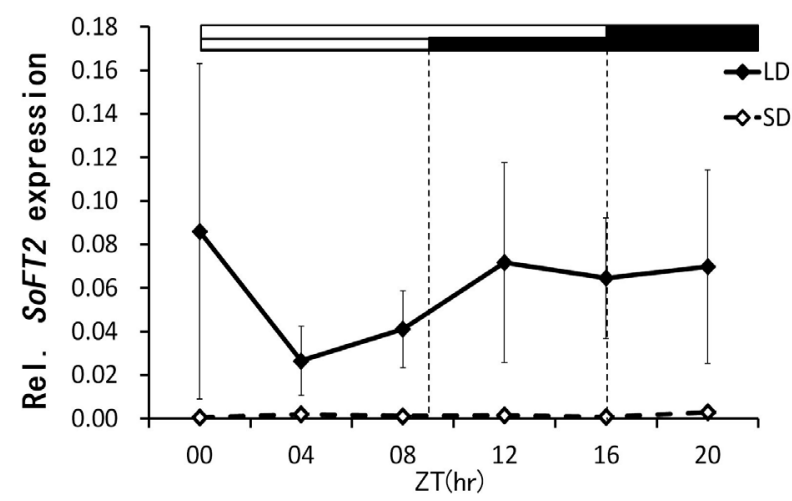

(c)

Figure 3. Diurnal variation of SoCOL1, SoFT1 and SoFT2 gene expressions under LD and SD conditions. Total RNA was extracted from seedlings at 3 to 4-leaf-stage every 4 h over a 24 h period, and expression levels of SoCOL1 (a), SoFT1 (b)

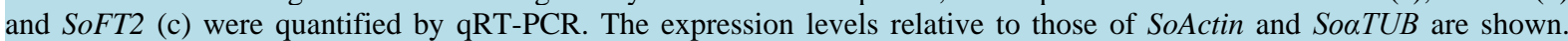
Error bars represent \pm SE from 4 independent experiments.

understand the correlation between expression patterns of SoFT1 and SoFT2 and flower bud formation, we performed quantitative RT-PCR analysis for tissue specific expression at different growth stages and microscopic observation of the shoot apex for flower bud formation. Based on the degree of leaf development and flower bud formation, we set three stages; stage I: seedlings had 1 - 2 developing true leaves and no flower buds (Figure 4(a) and Figure 4(d)), stage II: seedlings had 3 - 4 true leaves and some seedlings had flower buds (Figure 4(b) and Figure 4(e)), stage III: seedlings had 5 - 6 true leaves and flower buds (Figure 4(c) and Figure 4(f)). Expression of SoFT2 was high in the cotyledon and low in shoot apex at all stages examined in this study. In true leaves, SoFT2 expression increased gradually along with the growth stage progress, and was the highest after flower bud formation (Figure 4(g)). Expression of SoFT1 was clearly detected in cotyledons only at stage II and in true leaves only at stage III, and was ambiguous in other tissues because of low expression and non-specific amplification (data not shown).

\subsection{Response of SoFT1 and SoFT2 Expression to SD to LD Transition}

To investigate whether expression of SoFT1 and SoFT2 could respond to the transition from SD to LD, plants were grown in SD conditions until the fifth-leaf stage, under which spinach remained in a vegetative growth stage (Figure 5(a)), and then transferred to LD conditions, under which spinach initiated reproductive growth (Figures 5(c)-(e)). After transfer, leaves were harvested every 2 days. Expression levels of SoFT1 increased 2-fold within 2 days after transfer (Figure 5(f)), and maintained higher expression for 6 days. The expression level of SoFT2 was more obvious with a 20-fold induction within 2 days (Figure $5(\mathrm{~g})$ ), and then decreased to approximately a 10 -fold level. We confirmed that flower buds formed in all plants after 6 days of transfer to LD. SoFT1 and SoFT2 expression in plants not transferred to LD conditions had low levels of expression. These results indicated that the expression of SoFT1 and SoFT2 were associated with photoperiod transition and flower bud formation. 

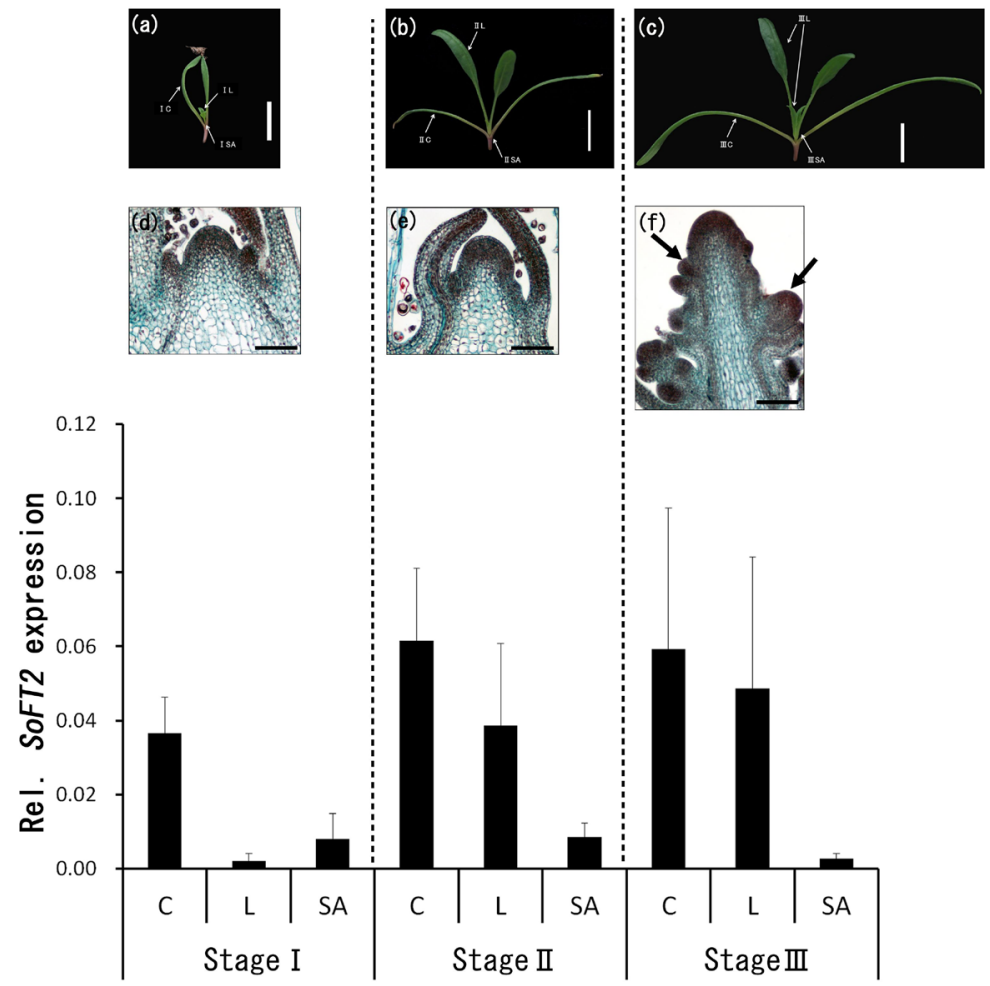

(g)

Figure 4. Flower bud formation and SoFT2 expression relative to development. Spinach seedlings were grown under LD conditions. At each developmental stage, stage I (a), stage II (b) and stage III (c), shoot apexes were observed by microscope to see flower bud formation (d, e and f, respectively). Black allow indicates flower primordia. White bars and black bars indicate $1 \mathrm{~cm}$ and $100 \mu \mathrm{m}$ respectively. Total RNA was extracted from cotyledons $(\mathrm{C})$, leaves (L) and shoot apex (SA) at ZT $=9$, and expression levels of SoFT2 were quantified by qRT-PCR (g). The expression levels relative to those of SoActin and So $\alpha T U B$ are shown. Error bars represent \pm SE from 5 independent experiments.

\section{Discussion}

It is very important to understand the mechanisms of reproductive phase (flowering and bolting) transition in spinach, because an undesirable transition is directly related to quality and yield loss. Although spinach requires a long day photoperiod for flowering and bolting, Oriental spinach, which is more desirable in Japan, is more sensitive to photoperiod than European spinach, and can be grown only from autumn to winter. In this study, to understand spinach flowering and bolting mechanisms at a genetic level, we isolated and characterized from Oriental spinach ("Nihon”), one CONSTANS-like (COL) and two FLOWERING LOCUS T (FT) homologs, which are known to be key factors of the flowering pathway in many plants.

SoCOL1 has two B-box and one CCT domains and belongs to Group I of the CONSTANS-like family together with AtCO, AtCOL1, AtCOL2 and BvCOL1 (Figure 1). The expression of the SoCOL1 gene showed a circadian rhythm with the highest expression at the end of the dark period in both LD and SD conditions (Figure 3(a)). These expression patterns are similar to BvCOL1, AtCOL1 and AtCOL2 [24], but opposite to AtCO. The expression of AtCO is induced by light, and activates FT transcription in Arabidopsis [7] [25]. Although AtCOL1 and AtCOL2 have been reported to have no effect on flowering time [24], over expression of BvCOL1 rescued the late-flowering phenotype of the Arabidopsis co-2 mutant, suggesting that BvCOL1 is functionally equivalent with AtCO [17]. SoCOL1 may be capable of inducing flowering, as is BvCOL1, because of high similarity of amino acid sequences and expression pattern. Recently, BBX19, a member of the B-box protein family, has been reported as a novel key component of flowering [26] [27]. In Arabidopsis, AtBBX19 interacts with At CO physically, and repress At FT transcription, resulting in delayed flowering [26]. In contrast to AtBBX19, BvBBX19 is thought to repress BvFT1 transcription and upregulate BvFT2 transcription, resulting in flower-promotion in sugar beet [27]. It is necessary to investigate whether a spinach BBX19 homolog interacts with SoCOL1 and 

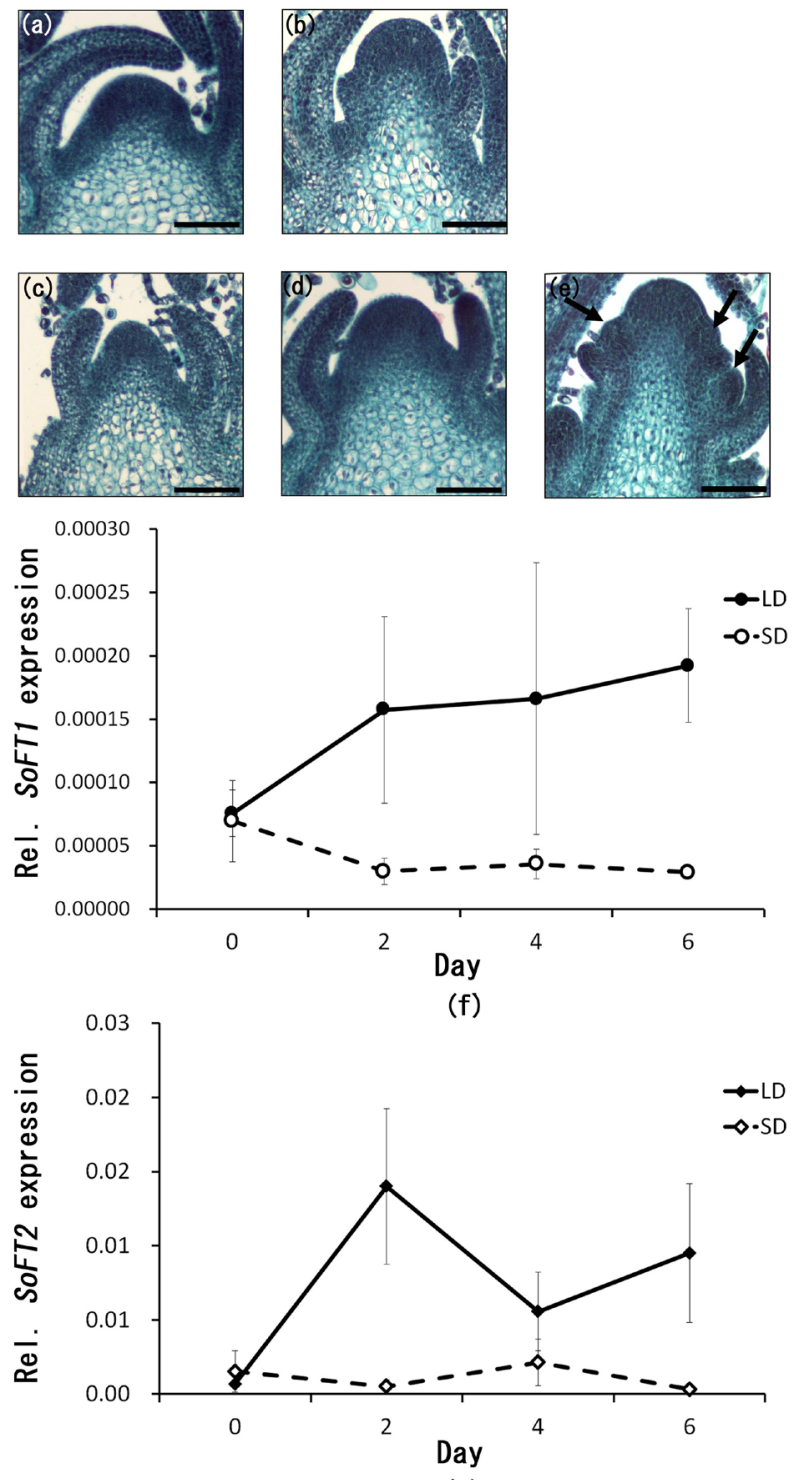

(g)

Figure 5. Flower bud formation and expression of SoFT1 and SoFT2 upon SD to LD transition. Spinach seedlings were grown in SD for around approximately one month, and then transferred to LD. After transfer to LD, shoot apexes were observed by microscope every 2 days. (a) before transfer, (b) 6 days without transfer, (c) 2 days after transfer, (d) 4 days after transfer, and (e) 6 days after transfer. Black allow indicates flower primordia. Black bars indicate $100 \mu \mathrm{m}$. Total RNA was extracted every 2 days, and SoFT1 and SoFT2 expression levels were quantified by qRT-PCR (f, and g, respectively).

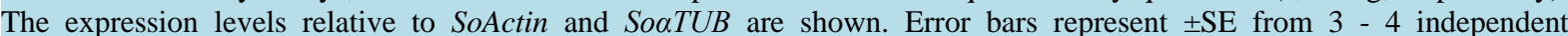
experiments.

affects the expression of SoFT1 and SoFT2.

From spinach, we isolated two FT homologs, SoFT1 and SoFT2, and revealed similarities and differences by comparison of amino acid sequences and expression analysis. SoFT2 conserves all functionally important amino acid residues reported thus far [22]-[24], and was classified as the closest homolog of BvFT2 [11] and CrFT1 [18] by phylogenetic analysis (Figure 2). The expression of SoFT2 was much higher when seedlings were grown under LD conditions compared to SD conditions (Figure 3(c)) with an increased expression becoming apparent within 2 days after transition from SD to LD (Figure 5(g)). SoFT2 was expressed in cotyledons and leaves under LD conditions and was not expressed in the shoot apex. Although the expression of SoFT2 did not show a clear diurnal rhythm (Figure 3(c)), SoFT2 may be a BvFT2 ortholog since most features are similar to 
BvFT2. On the other hand, the expression of SoFT1 was very low compared to the expression of SoFT2, and barely detectable in young seedlings and shoot apical meristems. The expression of SoFT1 was much higher in leaves from LD grown seedlings than in leaves from SD grown seedlings, showed a diurnal rhythm, and responded to the transition from SD to LD (Figure 3(b) and Figure 5(f)). Although SoFT1 as well as CrFT2 from Chenopodium rubrum were classified as the closest homologs of BvFT1 by phylogenic analysis, Gln-140 of AtFT, the conserved amino acid residue among the FT-like clade of the PEBP family, are substituted by Pro and Ile, respectively (Figure 2(a)). The Gln-140 in AtFT is located in the external loop at the entrance to the ligand-binding site, and interacts with Tyr-85 in AtFT, which is located in the ligand-binding site [22]. It has been reported that substitution of Gln-140 affected AtFT activity [21]. Furthermore, unlike BvFT1, SoFT1 and CrFT2 have three conserved amino acid residues, Tyr-134, Gly-137 and Trp-138 in BvFT2, which are crucial for flower-promoting activity. In BvFT1, a flowering repressor of sugar beet, these amino acid residues are substituted by Asn, Gln and Gln, respectively. Mutual exchanges of these residues between BvFT1 and BvFT2 converted their activity [11]. It would be interesting to know how these amino acid features affect SoFT1 and CrFT2 activities.

\section{Conclusion}

SoCOL1 and SoFT2 may contribute to spinach flowering regulation based on the similarity of amino acid sequences and expression patterns to BvCOL1 [17] and BvFT2 [11], respectively, although more experiments on protein levels will be necessary to clarify this point. In addition, our work found that spinach SoFT1 has the unusual substitutions of functionally important amino acid compared with other homologs belonging to the FT-like clade. These substitutions are similar to those of CrFT2 of C. rubrum [18], which is the most closely related plant species to spinach. It will be of interest to investigate whether spinach and C. rubrum evolved new FT homologs carrying a distinct function. In addition to further clarification of the physiological roles of SoCOL1, SoFT1 and SoFT2 in flowering and bolting, comparison of expression patterns of these genes between European and Oriental spinaches will provide a new strategy for spinach breeding.

\section{Acknowledgements}

We appreciate the DNA sequencing facility of the Graduate School of Agriculture, Hokkaido University, and Mr. Gregory Pearce for critical reading and suggestions on this manuscript. Financial support was provided partly by the Special Coordination Fund for accelerated innovation of the fostering system for female scientists, through the Japanese Ministry of Education, Culture, Sports, Science \& Technology's project (YY).

\section{References}

[1] Kagawa, A. (1974) Horenso: Nougyougijutsutaikei. Nobunkyou, Tokyo, Vol. 7, 3-8.

[2] Zeevaart, J.A. (2008) Leaf-Produced Floral Signals. Current Opinion in Plant Biology, 11, 541-547. http://dx.doi.org/10.1016/j.pbi.2008.06.009

[3] Song, Y.H., Ito, S. and Imaizumi, T. (2013) Flowering Time Regulation: Photoperiod- and Temperature-Sensing in Leaves. Trends in Plant Science, 18, 575-583. http://dx.doi.org/10.1016/j.tplants.2013.05.003

[4] Ballerini, E.S. and Kramer, E.M. (2011) In the Light of Evolution: A Reevaluation of Conservation in the CO-FT Regulon and Its Role in Photoperiodic Regulation of Flowering Time. Frontiers in Plant Science, 2, 81. http://dx.doi.org/10.3389/fpls.2011.00081

[5] Griffiths, S., Dunford, R.P., Coupland, G. and Laurie, D.A. (2003) The Evolution of CONSTANS-Like Gene Families in Barley, Rice, and Arabidopsis. Plant Physiology, 131, 1855-1867. http://dx.doi.org/10.1104/pp.102.016188

[6] Valverde, F. (2011) CONSTANS and the Evolutionary Origin of Photoperiodic Timing of Flowering. Journal of Experimental Botany, 62, 2453-2463. http://dx.doi.org/10.1093/jxb/erq449

[7] Tiwari, S.B., Shen, Y., Chang, H.C., Hou, Y., Harris, A., Ma, S.F., McPartland, M., Hymus, G.J., Adam, L., Marion, C., Belachew, A., Repetti, P.P., Reuber, T.L. and Ratcliffe, O.J. (2010) The Flowering Time Regulator CONSTANS Is Recruited to the FLOWERING LOCUS T Promoter via a Unique cis-Element. New Phytologist, 187, 57-66. http://dx.doi.org/10.1111/j.1469-8137.2010.03251.x

[8] Yamaguchi, A., Kobayashi, Y., Goto, K., Abe, M. and Araki, T. (2005) TWIN SISTER OF FT (TSF) Acts as a Floral Pathway Integrator Redundantly with FT. Plant and Cell Physiology, 46, 1175-1189. 
http://dx.doi.org/10.1093/pcp/pci151

[9] Abe, M., Kobayashi, Y., Yamamoto, S., Daimon, Y., Yamaguchi, A., Ikeda, Y., Ichinoki, H., Notaguchi, M., Goto, K. and Araki, T. (2005) FD, a bZIP Protein Mediating Signals from the Floral Pathway Integrator FT at the Shoot Apex. Science, 309, 1052-1056. http://dx.doi.org/10.1126/science.1115983

[10] Kobayashi, Y., Kaya, H., Goto, K., Iwabuchi, M. and Araki, T. (1999) A Pair of Related Genes with Antagonistic Roles in Mediating Flowering Signals. Science, 286, 1960-1962. http://dx.doi.org/10.1126/science.286.5446.1960

[11] Pin, P.A., Benlloch, R., Bonnet, D., Wremerth-Weich, E., Kraft, T., Gielen, J.J. and Nilsson, O. (2010) An Antagonistic Pair of FT Homologs Mediates the Control of Flowering Time in Sugar Beet. Science, 330, 1397-1400. http://dx.doi.org/10.1126/science.1197004

[12] Zeevaart, J.A. (1971) Effects of Photoperiod on Growth Rate and Endogenous Gibberellins in the Long-Day Rosette Plant Spinach. Plant Physiology, 47, 821-827. http://dx.doi.org/10.1104/pp.47.6.821

[13] Wu, K., Li, L., Gage, D.A. and Zeevaart, J.A. (1996) Molecular Cloning and Photoperiod-Regulated Expression of Gibberellin 20-Oxidase from the Long-Day Plant Spinach. Plant Physiology, 110, 547-554. http://dx.doi.org/10.1104/pp.110.2.547

[14] Lee, D.J. and Zeevaart, J.A. (2002) Differential Regulation of RNA Levels of Gibberellin Dioxygenases by Photoperiod in Spinach. Plant Physiology, 130, 2085-2094. http://dx.doi.org/10.1104/pp.008581

[15] Lee, D.J. and Zeevaart, J.A. (2005) Molecular Cloning of $G A_{2}$-Oxidase3 from Spinach and Its Ectopic Expression in Nicotiana sylvestris. Plant Physiology, 138, 243-254. http://dx.doi.org/10.1104/pp.104.056499

[16] Lee, D.J. and Zeevaart, J.A. (2007) Regulation of Gibberellin 20-Oxidase1 Expression in Spinach by Photoperiod. Planta, 226, 35-44. http://dx.doi.org/10.1007/s00425-006-0463-1

[17] Chia, T.Y., Müller, A., Jung, C. and Mutasa-Göttgens, E.S. (2008) Sugar Beet Contains a Large CONSTANS-LIKE Gene Family Including a CO Homologue That Is Independent of the Early-Bolting $(B)$ Gene Locus. Journal of Experimental Botany, 59, 2735-2748. http://dx.doi.org/10.1093/jxb/ern129

[18] Chab, D., Kolar, J., Olson, M.S. and Storchova, H. (2008) Two FLOWERING LOCUS T (FT) Homologs in Chenopodium rubrum Differ in Expression Patterns. Planta, 228, 929-940. http://dx.doi.org/10.1007/s00425-008-0792-3

[19] Drabešová, J., Cháb, D., Kolař, J., Haškovcová, K. and Štorchová, H. (2014) A Dark-Light Transition Triggers Expression of the Floral Promoter CrFTL1 and Downregulates CONSTANS-Like Genes in a Short-Day Plant Chenopodium rubrum. Journal of Experimental Botany, 65, 2137-2146. http://dx.doi.org/10.1093/jxb/eru073

[20] Kawade, K., Ishizaki, T. and Masuda, K. (2008) Differential Expression of Ribosome-Inactivating Protein Genes during Somatic Embryogenesis in Spinach (Spinacia oleracea). Physiologia Plantarum, 134, 270-281. http://dx.doi.org/10.1111/j.1399-3054.2008.01129.x

[21] Hanzawa, Y., Money, T. and Bradley, D. (2005) A Single Amino Acid Converts a Repressor to an Activator of Flowering. Proceedings of the National Academy of Sciences of the United States of America, 102, 7748-7753. http://dx.doi.org/10.1073/pnas.0500932102

[22] Ahn, J.H., Miller, D., Winter, V.J., Banfield, M.J., Lee, J.H., Yoo, S.Y., Henz, S.R., Brady, R.L. and Weigel, D. (2006) A Divergent External Loop Confers Antagonistic Activity on Floral Regulators FT and TFL1. The EMBO Journal, 25, 605-614. http://dx.doi.org/10.1038/sj.emboj.7600950

[23] Ho, W.W. and Weigel, D. (2014) Structural Features Determining Flower-Promoting Activity of Arabidopsis FLOWERING LOCUS T. Plant Cell, 26, 552-564. http://dx.doi.org/10.1105/tpc.113.115220

[24] Ledger, S., Strayer, C., Ashton, F., Kay, S.A. and Putterill, J. (2001) Analysis of the Function of Two CircadianRegulated CONSTANS-LIKE Genes. The Plant Journal, 26, 15-22. http://dx.doi.org/10.1046/j.1365-313x.2001.01003.x

[25] Suarez-Lopez, P., Wheatley, K., Robson, F., Onouchi, H., Valverde, F. and Coupland, G. (2001) CONSTANS Mediates between the Circadian Clock and the Control of Flowering in Arabidopsis. Nature, 410, 1116-1120. http://dx.doi.org/10.1038/35074138

[26] Wang, C.Q., Guthrie, C., Sarmast, M.K. and Dehesh, K. (2014) BBX19 Interacts with CONSTANS to Repress FLOWERING LOCUS T Transcription, Defining a Flowering Time Checkpoint in Arabidopsis. Plant Cell, 21, 3416 3420.

[27] Dally, N., Xiao, K., Holtgräwe, D. and Jung, C. (2014) The B2 Flowering Time Locus of Beet Encodes a Zinc Finger Transcription Factor. Proceedings of the National Academy of Sciences of the United States of America, 111, 1036510370. http://dx.doi.org/10.1073/pnas.1404829111 
Scientific Research Publishing (SCIRP) is one of the largest Open Access journal publishers. It is currently publishing more than 200 open access, online, peer-reviewed journals covering a wide range of academic disciplines. SCIRP serves the worldwide academic communities and contributes to the progress and application of science with its publication.

Other selected journals from SCIRP are listed as below. Submit your manuscript to us via either submit@scirp.org or Online Submission Portal.
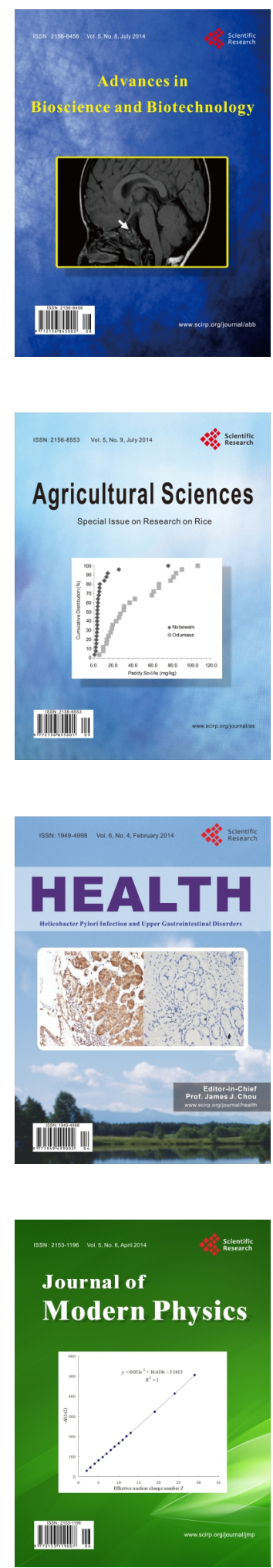
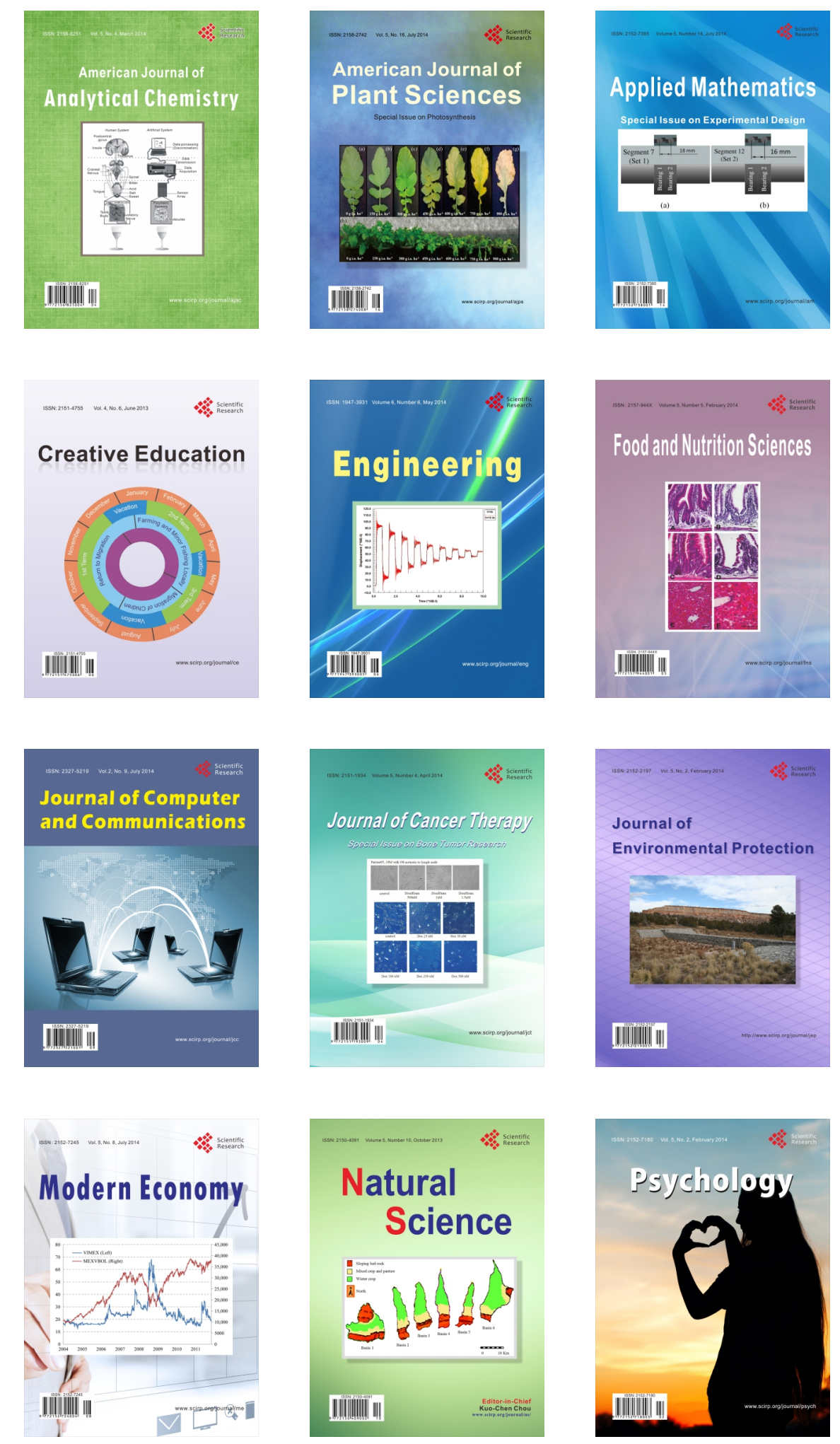